\title{
Analysis and design of a composite left-/right-handed leaky wave antenna based on the $H_{10}$ rectangular waveguide mode
}

\author{
Y. Weitsch and T. F. Eibert \\ Institute of Radio Frequency Technology, Universität Stuttgart, 70550 Stuttgart, Germany
}

\begin{abstract}
The presented "open" composite lefthanded/right-handed (CRHLH) substrate integrated waveguide performs well as a low-profile leaky wave antenna. This design is distinguished due to the fact that it is derived from the approved equivalent circuit model of the $H_{10}$ rectangular hollow waveguide mode. The wave propagation behaviour is visualised by the dispersion diagram calculated by two different methods, infinite periodic full-wave simulation and Matrix-Pencil analysis of driven field solutions. The periodic configuration is also analysed in terms of the Bloch impedance. Although FR-4 serves as substrate the antenna features an efficiency of about $50 \%$ to $60 \%$. The radiation performance demonstrates nearly backfire to almost endfire scanning capability of the antenna by mere frequency variation. Broadside radiation is possible due to the balanced state at $4 \mathrm{GHz}$.
\end{abstract}

\section{Introduction}

The presented leaky-wave antenna is not only attractive as conformal antenna. Having a low profile, it achieves a relatively high gain due to its large aperture. Leaky-wave antennas belong to the traveling wave type. The wave while propagating along the antenna structure radiates a certain part of the transported energy more or less in a continuous manner. The radiation direction is controllable by mere variation of frequency. Hence, neither additional phase shifters nor other complex feeding techniques are necessary. In terms of conventional right-handed $(\mathrm{RH})$ configurations, only endfire radiation is feasible. This restriction has been relieved with the discovery of metamaterials. In particular, combined righthanded/left-handed (CRHLH) (Lai and Itoh, 2004; Caloz

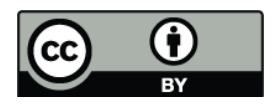

Correspondence to: Y. Weitsch (mail@ihf.uni-stuttgart.de) and Itoh, 2005) structures offer the possibility of radiation into the entire upper hemisphere. Additionally, the naturally occurring parasitic effects (of right-handed nature) are also taken into account as they are not suppressible. A peculiarity is broadside radiation resulting from the antenna being in the so-called balanced state. Figure 1 depicts the equivalent circuit of a combined RH/LH configuration. While the equivalent circuit (EQC) of a RH transmission line is well known, the one for the LH case is obtained by duality. A LH system means that the phase and the group velocity are antiparallel. Both EQCs are valid for infinitesimal lengths $\Delta z$ or rather if the line can be treated as uniformly homogeneous. For the same reason, a metamaterial can only be defined as such if its unit sections are much smaller than the wavelength of operation. Then, it deserves the name metamaterial with effective medium parameters disclosing novel characteristics on the macroscopic level. In the microscopic view, one unit section is normally repeated periodically facilitating the production process. The same holds for the dispersion analysis of the configuration which can be restricted to the examination of the irreducible Brillouin zone under the constraint of periodic boundary conditions. The total composition can be evaluated according to Floquet's theorem (Collin, 1991).

One of the common approaches to realise the desired properties in a metamaterial is to employ lumped or distributed circuit elements (Eleftheriades et al., 2002) to set up the EQC. Short-circuited or open transmission lines may also serve as the required inductors and capacitors. Conversely, this work begins with the established EQC given in Fig. 2 of the $H_{10}$ rectangular hollow waveguide mode (Fig. 3) (Marcuvitz, 1951). Important is that this EQC is very close to the aimed EQC of the CRHLH configuration since only the series capacitors are missing. Hence, only a small modification is sufficient to achieve the desired structure. One implementation is a rectangular waveguide with corrugated side walls like analysed in Eshrah and Kishk (2006). In order to simplify the production of the waveguide and being cost 


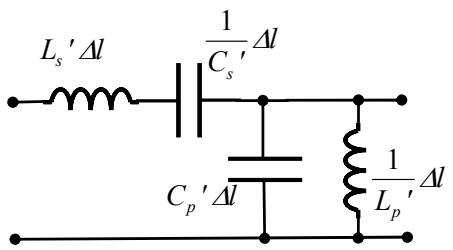

Fig. 1. Equivalent circuit model of a short section $\Delta l$ of a composite $\mathrm{LH} / \mathrm{RH}$ transmission line.

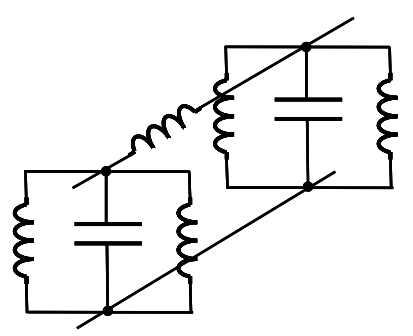

Fig. 2. Equivalent circuit model of the $H_{10}$ rectangular hollow waveguide mode.

effective, we utilise a substrate-integrated waveguide (SIW) (Deslandes and $\mathrm{Wu}, 2005$ ) in which the vertical side walls are replaced by rows of vias. Yet we aim at an "open" configuration with spurious radiation enabling the design of a composite RH/LH leaky-wave antenna.

In the following, we elucidate the design steps towards an ultra-wideband leaky-wave antenna with ample beam scanning capability including broadside direction. The description of the antenna involves an explanation of the balancing process precondition for broadside radiation. Likewise we attain matching. Since the antenna is constructed periodically, an analysis of the Bloch impedance characteristic for periodic configurations follows. The wave nature is investigated and illustrated by the dispersion diagram validated by two separate techniques, full-wave simulation of the infinite periodic configuration and the Matrix-Pencil estimation technique. The latter is applied to the field distribution of the gained driven solution. The measurements of a prototype reinforce the simulation results obtained by CST Microwave Studio.

\section{Antenna description}

Starting point is the EQC illustrated in Fig. 2 of the $H_{10}$ rectangular waveguide mode as depicted in Fig. 3. The lacking series capacitor may be implemented by slots in the top metallic plate perpendicular to the direction of the propagating wave. In contrast to conventional slotted waveguide designs, the slots are distanced much smaller than the wavelength not to influence higher order modes but to interact

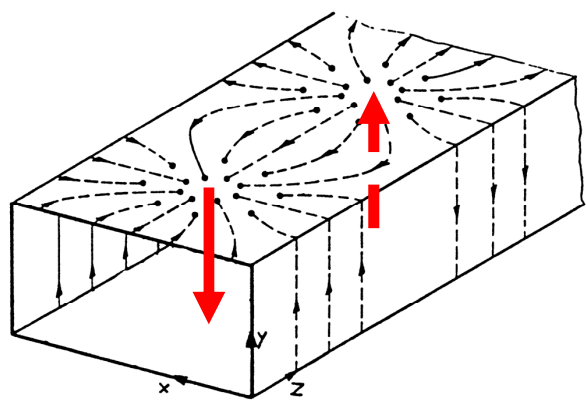

Fig. 3. Illustration of surface wall and displacement currents of the $H_{10}$ rectangular hollow waveguide mode.

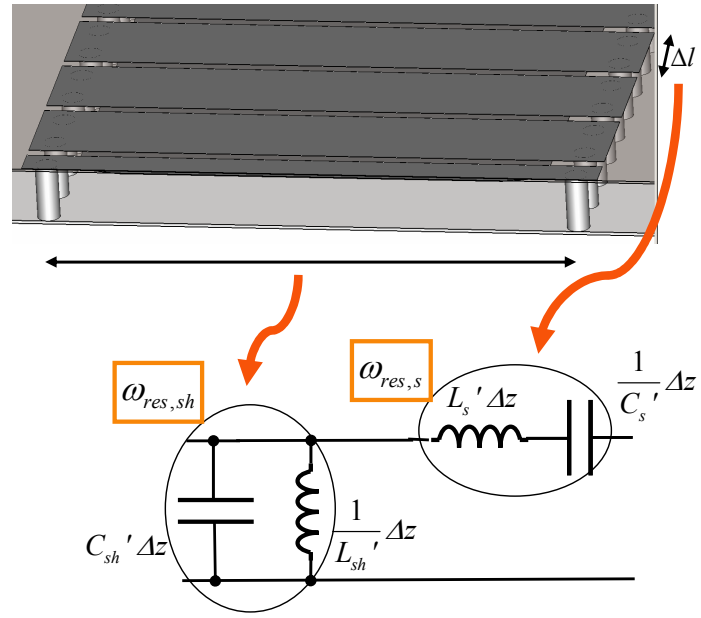

Fig. 4. Correspondence of SIW geometries and circuit model equivalences.

with the first mode able to propagate. One cut is very narrow to achieve a considerable capacitance value. A second level of metallic strips just below the upper metallization bridges the gaps and enhances the capacitance and we arrive at especially short sections (Figs. 4 and 5). Kapton foil acts as separation layer. The upper metallic bars are short-circuited with the metallic ground plane by via holes which now define the transverse dimensions of the waveguide. The horizontal geometry of the SIW not only prescribes the cut-off frequency of the original waveguide below which effective propagation was impossible. It also fixes the value of the shunt resonance of the EQC. The proportions in longitudinal direction determine the series resonance. For this reason, finding the balanced condition is very convenient since the important design parameters are decoupled from each other. So, balancing is typically achieved by adapting the length $\Delta l$ of one unit cell and the overlapping area covered by the metallic bars on the top and the metallic tongue slightly below (Figs. 4 and 5). Having reached the balanced state, 

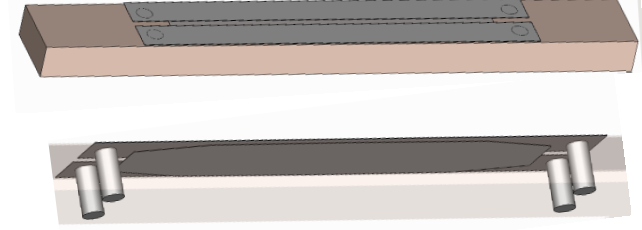

Fig. 5. Elementary cell of the periodic architecture.

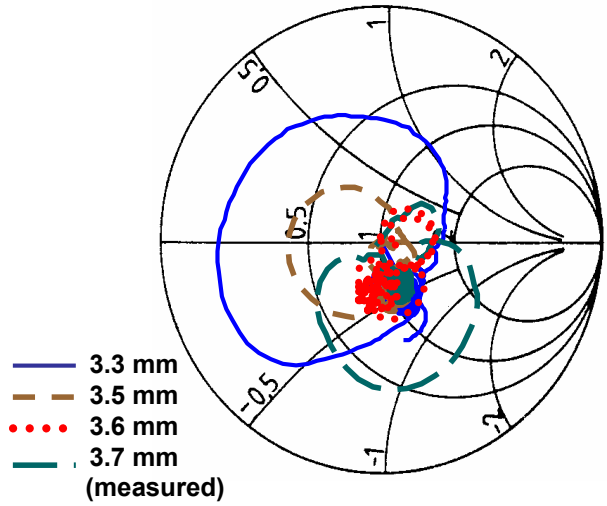

Fig. 6. $S_{11}$ of structures with different unit cell size lengths.

the series resonant frequency equates the resonant frequency of the shunt circuit or, if referring to a transmission line the characteristic impedance as issued in Caloz and Itoh (2005), ideally becomes independent from frequency. The effect of varying the length $\Delta l$ is visible in the Smith Chart shown in Fig. 6. Broadband matching is observed with a length of $3.6 \mathrm{~mm}$ as the curve of the reflection coefficient concentrates well around the matching point (red dotted line). This is moreover demonstrated with the magnitude of the input reflection coefficient shown in Fig. 7. The tapered transition section connecting the periodic cell configuration to a $50 \Omega$ microstrip line is thereby included. The final prototype is pictured in Fig. 8 being composed of 50 periods.

\section{Bloch impedance}

Since the traits in a periodic structure are repeated periodically with $p$, e.g along z-direction, the solution of the corresponding eigenvalue problem $\psi(z)$ can be represented as the product of a periodic function $\psi_{p}(z)$ and a propagation factor $\mathrm{e}^{-\gamma p}$ according to Bloch theorem:

$\psi(z)=\psi_{p}(z) \mathrm{e}^{-\gamma p} \quad$ with $\quad \psi_{p}(z+p)=\psi_{p}(z)$.

From an electromagnetic point of view, $\psi$ may describe the magnetic or the electric field in the strucuture. In order to

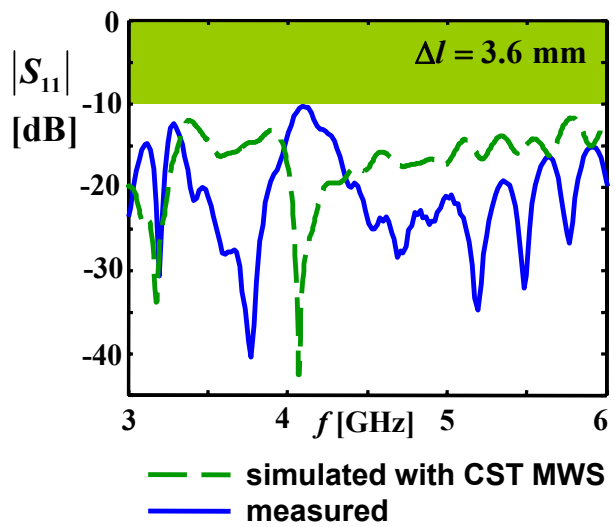

Fig. 7. $S_{11}$ of the LH / RH transmission line.

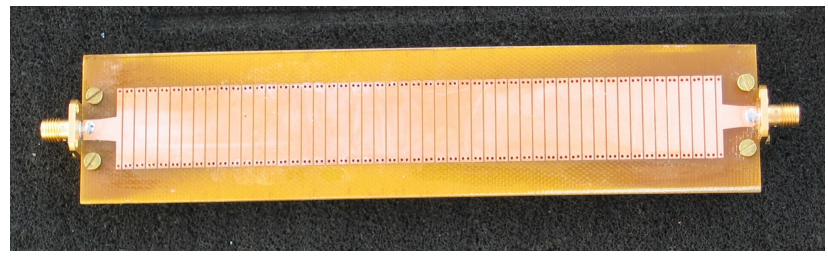

Fig. 8. Fabricated leaky-wave antenna.

gain an equivalent circuit model, it is required to find equivalent voltages and currents representing the waves in the structure. A possible definition of voltage $V$ and current $I$ is given by

$$
\begin{aligned}
V & =\int_{C_{v}} \boldsymbol{E} \cdot \boldsymbol{n}_{\text {ground }} d s, \\
I & =\int_{C_{i}}\left(\boldsymbol{n}_{g} \times \boldsymbol{H}\right) \cdot \boldsymbol{n}_{\text {port }} d s,
\end{aligned}
$$

according to a cross section as depicted in Fig. 9. Since these definitions are kind of arbitrary for non-TEM (transverseelectromagnetic)-waves (Marcuvitz, 1951), it is useful to also consider the power $P$ transported by the wave along the waveguide, which can be computed via

$$
P=\frac{1}{2} \iint_{S}\left(\boldsymbol{E} \times \boldsymbol{H}^{*}\right) \cdot \boldsymbol{n}_{\text {port }} d S \text {. }
$$

The required field data is extractable from the driven simulation. Based on these definitions, an characteristic impedance, the so-called Bloch impedance $Z_{B}$ (Collin, 1991), can be calculated on three different ways:

$$
Z_{B, P I}=\frac{2 P}{|I|^{2}}, \quad Z_{B, P V}=\frac{|V|^{2}}{2 P}, \quad Z_{B, V I}=\frac{V}{I} .
$$




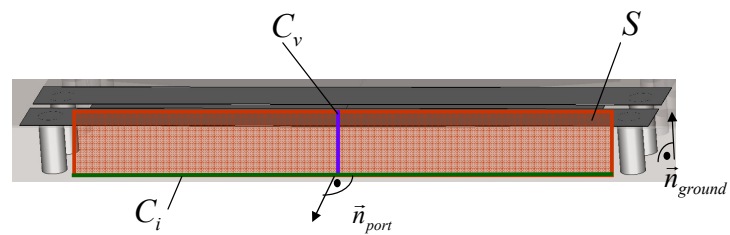

Fig. 9. Cross section within elementary cell.

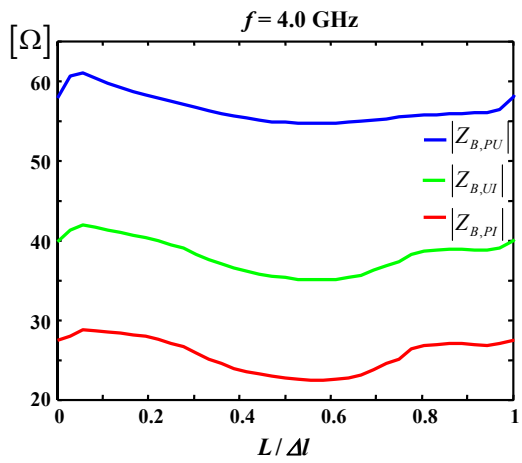

Fig. 10. Bloch impedance calculated along unit cell length according to the three definitions.

For TEM-waveguides, where the transverse fields are gradient fields, it is known that these three definitions give the same result for the characteristic impedance. However, for our case of a non-TEM-waveguide different results are obtained, where $Z_{B, V I}$ will not even lead to an equivalent circuit model in which the same power is transported as in the original waveguide (see Fig. 10). Similarly, different equivalent circuit models will be obtained, all describing the propagation behaviour of the considered wave, if the definitions remain consistent within the equivalent circuit. Since real power flow together with the longitudinal surface wall current components appear as physically most self-evident quantities, an characteristic impedance according to $Z_{B, P I}$ is usually preferred.

The Bloch impedance is identical at each periodic terminal plane. Since the location of a terminal can be set arbitrarily within one period, also considered as unit cell, it varies therein and this is the difference between the characteristic impedance of a homogeneous waveguide and the Bloch impedance in a waveguide composed of periodically repeated inhomogeneous unit cells. In Fig. $11, Z_{B}$ is calculated over frequency at the input interface. It is noted that the imaginary part changes from positive to negative values at about $4 \mathrm{GHz}$ around the transition frequency.

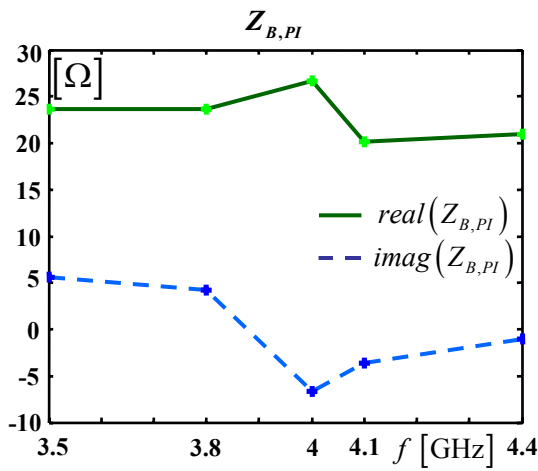

Fig. 11. Complex Bloch impedance.

\section{Dispersion diagram and radiation performance}

Together with the Bloch impedance, the propagation constant $\gamma=\alpha+j \beta$ sufficiently describes the eigenmode. Its imaginary part $\beta$, as solution of the eigenvalue equation or of the characteristic polynomial, is obtained by two techniques, the full-wave simulation of the infinite periodic configuration (by Ansoft HFSS) and from the field distribution of the driven problem by the Matrix-Pencil estimation method (Yingbo and Sarkar, 1990). The latter assumes a parametric signal model and approximates the field solution along a straight curve in propagation direction by a series of complex exponentials representing the various modes in the waveguide:

$y_{k}=x_{k}+n_{k}=\sum_{t=1}^{M} A_{t} \mathrm{e}^{\gamma_{t} \kappa k}+n_{k}$

The field samples $y_{k}$ are retrieved along the line from the field solution with the sample distance $\kappa$, while they may be overlayed by noise $n_{k}$. Subsequently, the so-called matrix pencils (special matrices) are formed helping to formulate an eigenvalue problem. Via singular value decomposition (SVD), the eigenvalues corresponding to the propagation constants are found and the modes having the strongest impact are filtered out. An estimation of the amplitudes follows according to the least mean square principle.

The obtained dispersion results are summarized in Figs. 12 and 13.

Both dispersion diagrams visualise the eigenfrequencies $f(\beta)$ belonging to the eigenvalues and verify the same dispersion behaviour. It is to emphasize that the antenna operates in the fundamental mode and not in higher space harmonics. If the eigenwave propagates in z-direction and attenuation along the waveguide is neglected, its spatial dependence in the far-field can be formulated by

$\psi(x, z) \sim \mathrm{e}^{-j \beta_{z} z} \mathrm{e}^{-k_{x} x}$

in which $k_{x}$ is the wavenumber component pointing in the air above and denotes radiation or attenuation, dependent on the 


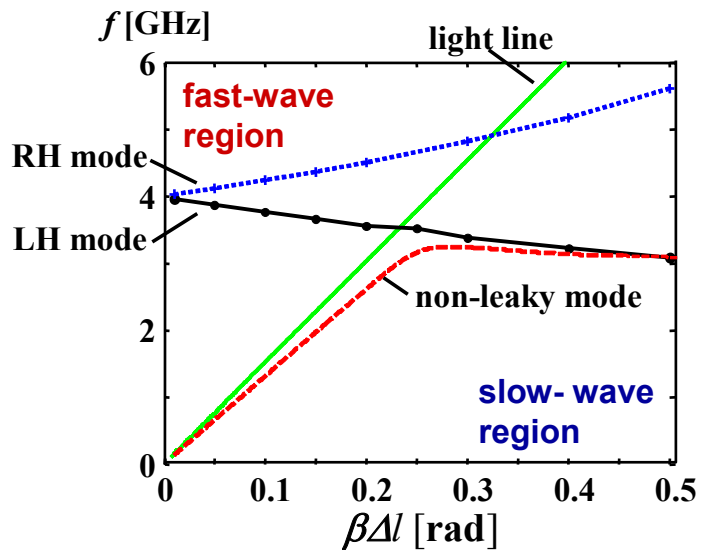

Fig. 12. Dispersion diagram computed by Ansoft HFSS.

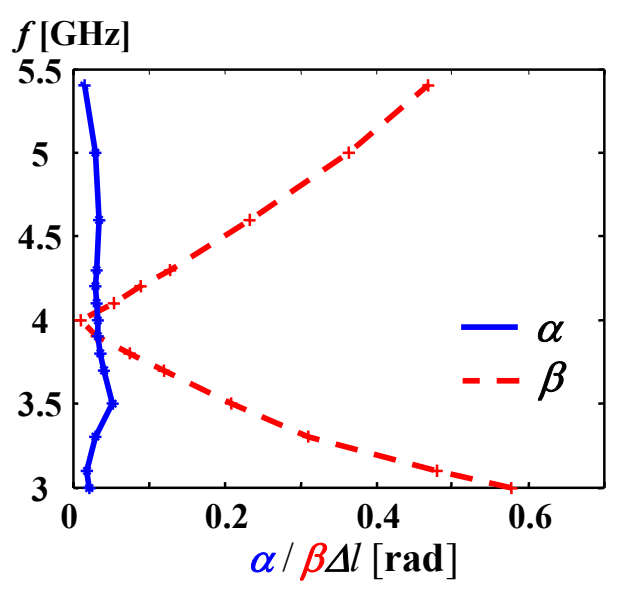

Fig. 13. Dispersion diagram obtained by the Matrix-Pencil method.

kind of solution to

$k_{x}=\sqrt{k_{0}^{2}-\beta_{z}^{2}}$

being real or imaginary. In the dispersion diagram in Fig. 12, the lowest mode found just represents a plane wave (nonleaky) traveling above the antenna structure. For increasing frequencies, the wave starts interaction with the waveguide to perceive in the bend-down of the curve. During its course, its $\beta_{z}$ values are larger than the phase constant of free space and thus no radiation in space occurs. The fundamental waveguide mode can be separated into two parts, a $\mathrm{LH}$ and a $\mathrm{RH}$ one. As anti-parallel group and phase velocity is exhibited only in the $\mathrm{LH}$ range, the slope of the curve belonging to the LH domain is negative. The upper twig of the curve is RH. Only in a balanced CRHLH structure like here, the RH mode continuously converts into the LH mode at the transition frequency, of about $4 \mathrm{GHz}$ in our case, with vanishing $\beta_{z}$ corresponding to infinite wavelength. If $\beta_{z}$ increases with rising

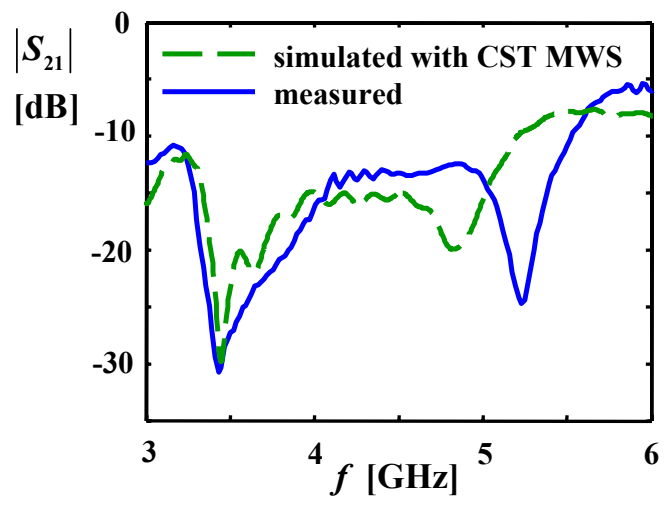

Fig. 14. Transmission factor for the $\mathrm{LH} / \mathrm{RH}$ transmission line.

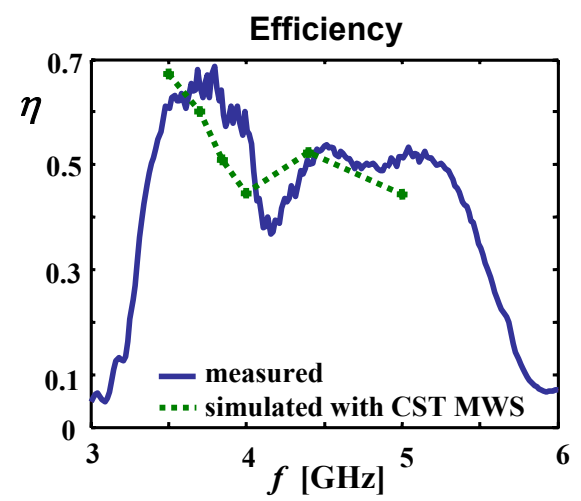

Fig. 15. Efficiency achieved with the dielectric FR-4.

frequency in the RH case and reducing frequency in the LH case, radiation in the upper hemisphere takes place as long as $\beta_{z}$ still remains less than the phase constant of free space and a real $k_{x}$ exists. Due to the anti-parallel feature in the LH domain, the phase constant points to the reverse direction resulting in a backfire beam. The endfire position is reached while the antenna is operated in the RH mode. Broadside radiation is only effective with $\beta_{z}=0$ and $k_{x}$ is identical with $k_{0}$. While $\beta_{z}$ further increases, it crosses the light line and becomes too large again in order to permit a real $k_{x}$. Consequently, the wave is solely guided again. In contrast to the dispersion diagram obtained by full-wave simulation, the estimation technique also reveals the attenuation constant $\alpha$. The attenuation of the wave along the waveguide not only represents the contribution of losses but also alludes to the amount of power leakage due to radiation. To shed light on the radiation performance, the transmission factor $S_{21}$ is given in Fig. 14. Its course proves broadband radiation as it is less than $-10 \mathrm{~dB}$ from about $3 \mathrm{GHz}$ up to $5.3 \mathrm{GHz}$. So, the accepted energy at the input is sufficiently consumed by leakage. To remark is the good accordance of the simulations and the measurements. Utilizing the relatively lossy substrate 


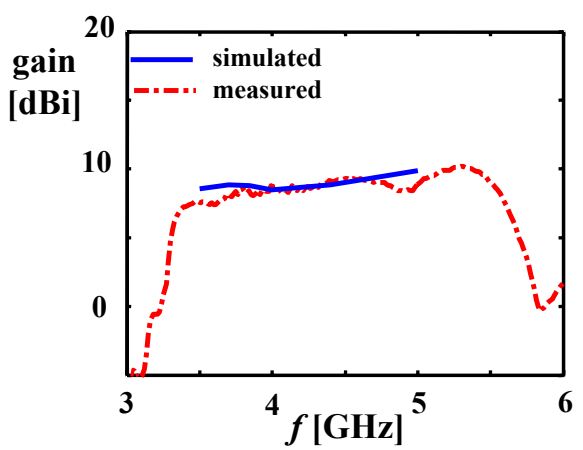

Fig. 16. Maximum gain in plane $\phi=0^{\circ}$ for each frequency.

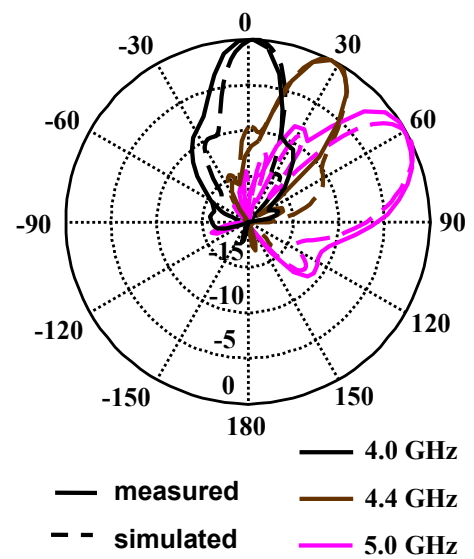

Fig. 17. Simulated and measured radiation patterns $[\mathrm{dB}]$ overlayed.

FR-4, an efficiency of $50 \%$ to $60 \%$ (Fig. 15) is attained. An augmentation in efficiency is conceivable by means of a low-loss substrate. Maximum gain is evaluated according to IEEE definition and illustrated in Fig. 16. For broadside radiation at $4 \mathrm{GHz}$, a gain of $8.64 \mathrm{dBi}$ is reached. The maximum gain of $10.15 \mathrm{dBi}$ is measured at $5.3 \mathrm{GHz}$, which corresponds to a scan angle of over $70^{\circ}$. In Fig. 17, several radiation pattern cuts stemming from measurements and from simulations are superimposed and reveal good agreement. The side lobes are roughly around $-10 \mathrm{~dB}$ with respect to the main lobe. Last but not least, in Fig. 18 the frequency-dependent scan behaviour is depicted verifying the contiguous radiation performance.

\section{Conclusions}

A broadband conformal leaky-wave antenna was presented which is continuously scannable from nearly endfire to backfire including broadside radiation. Its efficiency reaches about $50 \%$ to $60 \%$ despite of the low-cost and lossy dielectric substrate FR-4. In contrast to other configurations,

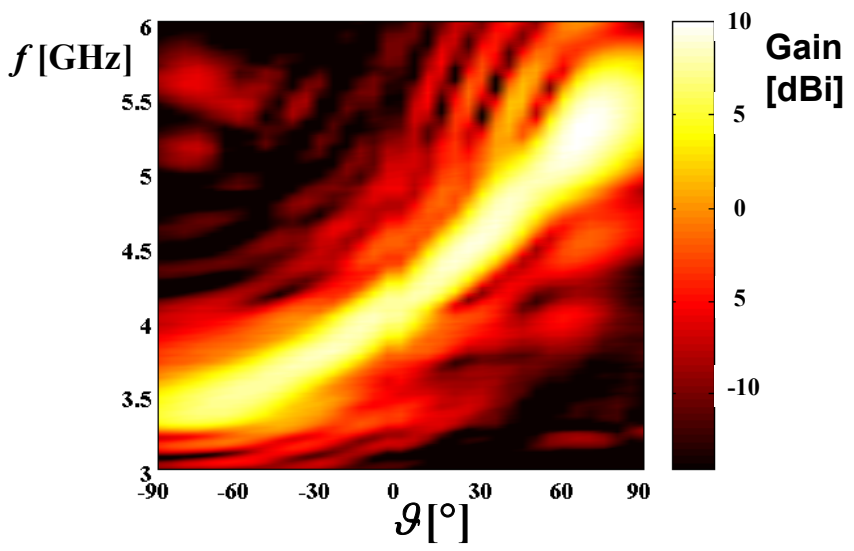

Fig. 18. Frequency-dependent scan performance from $-90^{\circ}$ to $+90^{\circ}$.

the presented design is based on the $H_{10}$ rectangular hollow waveguide mode, which was upgraded to a composite left-/righ-handed mode by introducing transverse slots with narrow spacing. It was optimised for broadside radiation at around $4 \mathrm{GHz}$. Simulation and measurement results are in good accordance and demonstrate good radiation behaviour and beam scanning capability of the leaky-wave antenna.

\section{References}

Caloz, C. and Itoh, T.: Electromagnetic Metamaterials: Transmission Line Theory and Microwave Applications, Wiley, New York, 2005.

Collin, R. E.: Field Theory of Guided Waves, 2nd ed., IEEE Press, New York, 1991.

Deslandes, D. and Wu, K.: Analysis and Design of Current Probe Transition From Grounded Coplanar to Substrate Integrated Rectangular Waveguides, IEEE Trans. Microwave Theory Techniques, 53(8), 2487-494, 2005.

Eleftheriades, G. V., Iyer, A. K., and Kremer, P. C.: Planar negative refractive index media using periodically L-C loaded transmission lines, IEEE Trans. Microwave Theory and Techniques, 50(12), 2702-2712, 2002.

Eshrah, I. A. and Kishk, A. A.: Analysis of Left-handed Rectangular Waveguides with Dielectric-filled Corrugations Using the Asymptotic Corrugation Boundary Conditions, IEE Proc.Microw. Antennas Propag., 153(3), 221-225, 2006.

Lai, A. and Itoh, T.: CompositeRight/Left-Handed Transmission Line Metamaterials, IEEE Microwave Mag., pp. 34-50, 2004.

Marcuvitz, N.: Waveguide Handbook, McGrall-Hill, New York, 1951.

Yingbo, H. and Sarkar, T. K.: Matrix Pencil Method for Estimating Parameters of Exponentially Damped/Undamped Sinusoids in Noise, IEEE Transactions on Acoustics, Speech and Signal Processing, 38(5), 1990. 